\title{
Study on the Impact of Polymer Concentration and Coagulation Bath Temperature on the Porosity of Polyethylene Membranes Fabricated Via TIPS Method
}

\author{
Ali Akbari ${ }^{1,2}$ and Reza Yegani ${ }^{1,2, *}$ \\ ${ }^{1}$ Faculty of Chemical Engineering, Sahand University of Technology, Sahand New Town, Post Box \\ 51335/1996, Tabriz, Iran \\ ${ }^{2}$ Membrane Technology Research Center, Sahand University of Technology, Sahand New Town, Post Box \\ 51335/1996, Tabriz, Iran
}

\begin{abstract}
Microporous high density polyethylene flat membranes were fabricated via thermally induced phase separation (TIPS) method. Effects of polymer concentration and coagulation bath temperature on the membrane morphology and porosity were investigated. To the best of our knowledge, there is no work about the order of magnitude and degree of importance of influential parameters and their interactions on the microstructure of fabricated membranes. The results showed that the porosity of membranes decreased as the polymer concentration increased. It was also shown that, due to the short contact time and rapid phase inversion between coagulation bath and membrane's outer surfaces, bath temperature mainly affects on the outer surface porosity. The results obtained from analysis of variance (ANOVA) using $95 \%$ confidence interval on the membrane porosity revealed that the effect of polymer concentration is more important than coagulation bath temperature.
\end{abstract}

Keywords: HDPE membrane, Experimental design, Analysis of variance (ANOVA), Leafy structure, Spherulitical structure.

\section{INTRODUCTION}

The last several decades have witnessed a tremendous growth of synthetic polymeric membranes as a tool for the separation the component from the mixture. The search for the elucidating mechanisms of membrane function makes it possible to utilize various membranes for sophisticated applications, such as in membranes for microfiltration, ultrafiltration, reverse osmosis, dialysis, and electrodialysis [1].

There are two main techniques for the preparation of polymeric membranes by phase separation. If phase separation is occurred by exchange of a non-solvent and solvent in polymer solution, the process is referred to as non-solvent induced phase separation (NIPS) or immersion precipitation. However, if the phase separation is induced by cooling the melt polymer solution, the process is referred to as thermally induced phase separation (TIPS) [2]. TIPS is one of the most useful techniques to prepare microporous polymer membrane and has been studied extensively for the fabrication of membrane using wide ranges of amorphous, crystalline and semi-crystalline polymers [3]. TIPS process includes two mechanisms; solidliquid (S-L) and liquid-liquid (L-L) phase separations

*Address corresponding to this author at the Faculty of Chemical Engineering, Sahand University of Technology, Sahand New Town, Post box: 51335/1996, Tabriz, Iran; Tel: (0098) 412-345-9147; Fax: (0098) 412-344-4355;

E-mail: ryegani@sut.ac.ir with subsequent crystallization [4]. Lloyd et al. studied solid-liquid [5] and liquid-liquid [6] phase separation of porous polymeric membranes prepared via TIPS method and investigated the effects of thermodynamic interactions [7], crystallization kinetics [8], properties of diluent including mobility, crystallization and morphology [9-10] and effect of dilution, cooling rate and nucleating agent addition [11] on the membrane structures. Variety of polymers such as polypropylene (PP), polystyrene (PS), poly vinylidene fluoride (PVDF), cellulose acetate (CA) and poly (ethylene-co-vinyl alcohol) (PE-VA) have been used for membrane fabrication through TIPS process [4]. In case of polyethylene (PE) membranes, Matsuyama et al. investigated the effect of $P E$ density and kinds of diluents on hollow fiber membranes [12, 13]. They illustrated that high density polyethylene (HDPE) membranes have larger pores and higher water permeability than low density polyethylene membranes (LDPE). Sun et al. illustrated that the water permeability of the membrane influenced by polymer melt flow index (MFI), liquid paraffin (LP) content and membrane thickness. They showed that the water permeability increases when the MFI and membrane thickness decrease. They also revealed that increasing of LP content increases the water permeability [14].

It has been reported that parameters like polymer molecular weight, polymer density, polymer-diluent ratio, coagulation bath temperature and type of 
extractant affect on the membranes structures and performances. Among these parameters, polymer concentration and coagulation bath temperature were selected as more affective parameters, since they directly affect on phase separation mechanism and membrane morphology [5].

Many studies have thus far been conducted regarding the fabrication of polyethylene membranes; however, no concrete results have been achieved about the mutual impact and significance of influential parameters. Experimental design is a branch of knowledge helping to measure the impact of influential factors $(X 1, X 2, X 3, \ldots)$ on the outputs $(Y 1, Y 2, Y 3, \ldots)$ under $\mathrm{Yi}=\mathrm{F}(\mathrm{Xi})$ equation [15]. It is commonly used to study and analysis the performance of processes and determination of key product design parameters that impact product performance. Examining the individual and mutual impacts of parameters in order to increase the membrane porosity was among the main objectives of this research. In statistical significance testing, the Pvalue is the probability of obtaining a test statistic at least as extreme as the one that was actually observed and F-value, indicatives of the significance of parameters, can meet this objective.

According to the number of levels using in this work, the best method for design of experiments (DOE) considering all of conditions thoroughly is the full factorial experimental design. This method was the best option to achieve the objective as it minimizes errors.

Generally, in a full factorial experimental design, experimental trials are performed at all combination of factor levels. When several factors are of interest in an experiment, a factorial experimental design should be used. In this method, the effect of the factor is defined as the change in response produced by a change in the level of the factor [15].

In this work, HDPE was used to prepare flat plat membranes. Liquid paraffin was selected as diluents, since it has a good compatibility with PE due to their similar chemical structures and close solubility parameters [3].

The objective of the present work was to study the impact of polymer concentration and cooling bath temperature on membrane porosity. Using full factorial design of experiments the individual and mutual interaction of influential parameters as well as their order of magnitude and the level of importance on the membrane porosity were determined. The impacts of those influential parameters were also considered on the structure and morphology of pores.

\section{EXPERIMENTAL}

\subsection{Materials}

High density polyethylene (HDPE) was provided by Tabriz Petrochemical Company (3840, MFI $=0.35$ $\mathrm{g} / 10 \mathrm{~min}, 190^{\circ} \mathrm{C}, 2.16 \mathrm{~kg}$ ). Liquid paraffin (extra pure), acetone (extra pure) and isobutanol (extra pure) were used as diluent, extracting solvent and porosity measurement agent, respectively were purchased from Merck. All chemicals used in this study were not further purified.

\subsection{Flat Plate Membrane Preparation}

To prepare flat plate membranes, specific amounts of HDPE and liquid paraffin were put into overhead mixer and warmed up to at least $20^{\circ} \mathrm{C}$ above the melting point of the polymer-diluent mixture to form a homogeneous solution. In order to measure the cloud point, the samples were heated on a hot stage (LEICA DMRX) up to $180^{\circ} \mathrm{C}$ for $3 \mathrm{~min}$ and then cooled to $25^{\circ} \mathrm{C}$ with a controlled rate of $1{ }^{\circ} \mathrm{C} / \mathrm{min}$. However, obtained results showed that for PE/LP system instead of cloud point, crystallization temperature is only existed. This finding was quite similar with the work performed by Matsuyama et al. [13].

Then the solution was poured on pre-heated glass plates and spread out using a casting knife and then immediately immersed into coagulation bath to induce phase separation. The obtained sample was detached from the glass and immersed in acetone overnight for $24 \mathrm{hr}$ to extract the diluents. To ensure the evaporation of extractant, samples were heated in a vacuum oven at $50^{\circ} \mathrm{C}$ for $2 \mathrm{hr}$. Finally, the microporous membrane was obtained.

\subsection{Membrane Porosity}

The resulted membranes were cut into small pieces. The porosity is determined by impregnating membranes with isobutanol for 24 hours. The impregnated samples were blotted with tissue papers to remove the residual isobutanol on the membranes. The mass of the samples before and after immersing in isobutanol measured using a digital microbalance. The porosity of the membrane $(\varepsilon)$ is defined as the pore volume divided by the total volume of the membrane as follows [17]: 
Table 1: Factors and Levels Used in Fabrication of Flat Sheet Membranes

\begin{tabular}{|c|c|c|c|}
\hline Factor & Type & Levels & Values \\
\hline \hline Polymer concentration (wt. \%) & Fixed & 3 & $15,20,25$ \\
\hline Water bath temperature (K) & Fixed & 3 & $273,303,333$ \\
\hline Total tests with 2 replications & & 18 & \\
\hline
\end{tabular}

$\varepsilon(\%)=\frac{\left(w_{w}-w_{d}\right) \times \rho_{p}}{\left(w_{w}-w_{d}\right) \times \rho_{p}+w_{d} \times \rho_{i}} \times 100 \%$

Where $w_{w}$ is the mass of the wet sample $(\mathrm{g}), w_{d}$ is the mass of the dry sample $(\mathrm{g}), \rho_{p}$ is the polyethylene density $\left(0.95 \mathrm{~g} / \mathrm{cm}^{3}\right)$ and $\rho_{i}$ is the isobutanol density $\left(0.802 \mathrm{~g} / \mathrm{cm}^{3}\right)$. The assumption of this method is that, all the pores were completely filled with isobutanol.

\subsection{Structural Characterization of Flat Plat Membranes}

Flat membranes were fractured in liquid nitrogen and treated with $\mathrm{Au} / \mathrm{Pd}$ sputtering. The cross-sections and the surfaces of prepared membranes were examined using a scanning electron microscope (CamScan MV2300).

\section{RESULTS AND DISCUSSION}

\subsection{Experimental Design}

HDPE membranes were prepared as follows as reported previously. In this work, HDPE membranes were prepared with 15,20 and $25 \%$ (w/w) of polymer concentration in liquid paraffin solution. Phase separation process was carried out in three different coagulation bath temperatures such as 0,30 and 60 ${ }^{\circ} \mathrm{C}$. The thickness of membranes was adjusted at 500 $\mu \mathrm{m}$ using casting knife. As shown in Table 1, 9 different membranes were fabricated in three levels of polymer concentrations and coagulation bath temperatures. To decrease the measurement errors all experiments were repeated twice therefore total number of experiments would be 18. Table 2 shows the obtained results of measured porosity and Table $\mathbf{3}$ lists the data variance analysis for porosity. Data in Tables $\mathbf{3}$ and $\mathbf{4}$ show the degree of freedom (Df), sum of squares of factors (Seq SS), adjusted sum of squares of levels (Adj SS), adjusted mean squares (Adj MS), $F$ and $P$ values. Although the detail calculation methods of mentioned parameters are given in text book [15] however, brief information and basic calculations are shown as follow.

The p-values $(P)$ in the analysis of variance table used to determine which of the effects in the model are statistically significant. If the p-value is lower than 0.05 , then the factor is significant.

The name of analysis of variance is derived from a partitioning of total variability into its component parts. Suppose we have a treatments or different levels of a factor. $y_{i j}$ represents the $j^{\text {th }}$ observation taken under factor level or treatment $i$. In general, there are $n$ observations (replicates) under the $I^{\text {th }}$ treatment.

Sum of squares represents a measure of variation or deviation from the mean value. The total corrected sum of squares represents by Eq. 2 as follow:

Table 2: Obtained Results from Analysis of Fabricated Membranes

\begin{tabular}{|c|c|c|}
\hline Concentration \% (w/w) & Water Bath Temperature (K) & Average Porosity (\%) \\
\hline \hline 15 & 273 & 74.2625 \\
\hline 15 & 303 & 77.92 \\
\hline 15 & 333 & 71.3625 \\
\hline 20 & 273 & 73.6225 \\
\hline 20 & 303 & 75.46 \\
\hline 20 & 333 & 68.83 \\
\hline 25 & 273 & 72.13 \\
\hline 25 & 303 & 73.855 \\
\hline 25 & 333 & \\
\hline
\end{tabular}


Table 3: Data of Analysis of Variance (ANOVA) for Membrane Porosity

\begin{tabular}{|c|c|c|c|c|c|c|}
\hline Source & DF & Seq SS & Adj SS & Adj MS & F \\
\hline \hline C & 2 & 140.792 & 140.792 & 70.396 & 35.44 & 0.000 \\
\hline Tk & 2 & 101.324 & 101.324 & 50.662 & 25.51 & 0.000 \\
\hline C $^{*}$ Tk & 4 & 10.860 & 10.860 & 2.715 & 1.37 & 0.319 \\
\hline Error & 9 & 17.876 & 17.876 & 1.986 & & \\
\hline Total & 17 & 270.851 & & & \\
\hline
\end{tabular}

Table 4: Data of Analysis of Variance (ANOVA) for Regression Equation

\begin{tabular}{|c|c|c|c|c|c|c|}
\hline Source & DF & Seq SS & Adj SS & Adj MS & F & P \\
\hline \hline Regression & 2 & 233.645 & 233.645 & 116.823 & 47.0989 & 0.000000 \\
\hline Lack-of-Fit & 6 & 19.330 & 19.330 & 3.222 & 1.6220 & 0.24667 \\
\hline Pure Error & 9 & 17.876 & 17.876 & 1.986 & & \\
\hline Total & 17 & 270.851 & & & \\
\hline
\end{tabular}

$\operatorname{Adj} \mathrm{SS}_{\mathrm{T}}=\sum_{\mathrm{i}=1}^{\mathrm{a}} \sum_{\mathrm{j}=1}^{\mathrm{n}}\left(\mathrm{y}_{\mathrm{ij}}-\overline{\mathrm{y}}_{\mathrm{n}}\right)$

Sum of squares is used as a measure of overall variability in the data. $\bar{y}_{\mathrm{n}}$ represents the grand average of all the observations.

Adjusted sum of squares is calculated as a summation of the squares of the differences from the mean value. The calculation of the adjusted total sum of squares considers both the adjusted sum of squares from the factors and from random chance or error. Thus, we may write:

$\operatorname{Adj} \mathrm{SS}_{\mathrm{T}}=\operatorname{Adj} \mathrm{SS}_{\text {Treatments }}+\operatorname{Adj} \mathrm{SS}_{\mathrm{E}}$

Where Adj $\mathrm{SS}_{\text {Treatments, }}$ which is shown by Eq. 4, is called the adjusted sum of squares due to treatment, and Adj $S_{E}$ is called the adjusted sum of squares due to error.

$\operatorname{Adj} \mathrm{SS}_{\text {Treatments }}=\mathrm{n} \sum_{\mathrm{i}=1}^{\mathrm{a}}\left(\overline{\mathrm{y}}_{\mathrm{i}}-\overline{\mathrm{y}}_{\mathrm{n}}\right)^{2}$

where

$\bar{y}_{\mathrm{i}}=\mathrm{y}_{\mathrm{i}} / \mathrm{n}, \mathrm{y}_{\mathrm{i}}=\sum_{\mathrm{j}=1}^{\mathrm{n}} \mathrm{y}_{\mathrm{ij}}$

Degree of freedom is the amount of information provided by the data that makes possible to "spend" to estimate the values of unknown population parameters, and calculate the variability of these estimates. There are $\boldsymbol{a} \times \boldsymbol{n}=\boldsymbol{N}$ total observations; thus, $\mathrm{SS}_{\mathrm{T}}$ has $\boldsymbol{N - 1}$ degrees of freedom. There are $\boldsymbol{a}$ levels of the factor, so $\mathrm{SS}_{\text {Treatments }}$ has $\boldsymbol{a}-\mathbf{1}$ degrees of freedom. Finally, within any treatment there are $n$ replicates providing $n-1$ degrees of freedom with which to estimate the experimental error. Because there are $\boldsymbol{a}$ treatments, we have $\mathrm{N}$ - $\boldsymbol{a}$ degree of freedom for error which can be calculated by the following Eq. 6 .

$a(n-1)=(a \times n)-a=N-a$

Minitab software which is used in this article, displays both the sequential sums of squares (Seq SS) and adjusted sums of squares (Adj SS). If the model is orthogonal and does not contain covariates, these will be the same.

The quantities of Adj $M_{\text {Treatments }}$ and Adj $M S_{E}$ which are shown by Eq. 7 and 8, respectively, are called mean squares.

Adj $\mathrm{MS}_{\text {Treatments }}=\frac{\operatorname{Adj} \mathrm{SS}_{\text {Treatments }}}{\mathrm{a}-1}$

$\operatorname{Adj} \mathrm{MS}_{\mathrm{E}}=\frac{\operatorname{Adj} \mathrm{SS}_{\mathrm{E}}}{\mathrm{N}-\mathrm{a}}$

Dividing the Adj $\mathrm{MS}_{\text {Treatments }}$ by the Adj $\mathrm{MS}_{\mathrm{E}}$ gives $\boldsymbol{F}$, which follows the $\boldsymbol{F}$ distribution with degrees of freedom for the term and degrees of freedom for error. $\boldsymbol{F}$ value is defined by the following Eq. 9:

$$
\mathrm{F}=\frac{\operatorname{Adj} \mathrm{SS}_{\text {Treatments }} /(\mathrm{a}-1)}{\operatorname{Adj~SS}_{\mathrm{E}} /(\mathrm{N}-\mathrm{a})}=\frac{\operatorname{Adj} \mathrm{MS}_{\text {Treatments }}}{\operatorname{Adj} \mathrm{MS}_{\mathrm{E}}}
$$


$\boldsymbol{F}$ is used to test whether the effect of a term in the model (factor or interaction) is significant.

The results obtained from analysis of variance table using 95 percent confidence interval on the membrane porosity illustrated that the impact of polymer concentration $(\mathrm{C})$ on the membrane porosity is more severe than the coagulation bath temperature (Tk). Interestingly the mutual impact of influential parameters on the membrane porosity is negligible.

The regression equation of the response porosity $(\mathrm{P})$ on the two significant independent variables $\mathrm{C}$ and Tk can be approximated by a nonlinear polynomial model. Equation 10 shows the general regression equation for porosity vs. polymer concentration (C) and water bath temperature (Tk).

$P=\beta_{0}+\beta_{1} C+\beta_{2} T k+\beta_{12} C^{*} T k$

Where, $P$ is membrane porosity, $\beta_{0}$ is the average of the all obtained data, $\beta_{1}, \beta_{2}$ and $\beta_{12}$ are the coefficients of polymer concentration, coagulation bath temperature and their mutual interaction, respectively. Since the mutual interaction of affecting parameters is negligible, the regression equation is simplified as shown in equation 11.

$\mathrm{P}=58.4015-0.664417 \times \mathrm{C}+0.0968056 \times \mathrm{Tk}$

In Eq. 11, the regression square (R-Sq) which measures of how well the model fits the data, equals to 86.26, which ensures the best fit of experimental results.

\subsection{Effect of Polymer Concentration on the Membrane Porosity}

As shown in Table 3, the impact of polymer concentration on the membrane porosity is more severe than coagulation bath temperature. This issue could be justified by the different influence of both parameters on the membrane porosity. Lloyd et al. showed that in solid-liquid phase separation, increasing polymer concentration, increases nucleation density, which in turn results in decreased porosity [5]. Since nucleation is mainly occurred in the bulk of polymer, it can be concluded that the polymer concentration mainly affects on the bulk porosity and inner structure of membrane. On the other hand, due to the short contact time and rapid phase inversion between coagulation bath and membrane's outer surface, bath temperature mainly affects on the outer surface porosity. Since in this work, the overall porosity is measured, the obtained results would mainly express the bulk porosity of the membrane.

Figure 1 shows the impact of polymer concentration on the membrane porosity. As illustrated in Figure 1, increasing of polymer concentration decreases the membrane porosity in all temperature domains. Reduction in porosity is mainly occurred in bulk porosity of membrane. This issue could be justified by the fact that increasing of the polymer concentration decreases the size of internal pores and the spaces between spherulites. Therefore the number of spherulites increases which results in higher nucleation density at higher polymer concentration and therefore membrane porosity decreases.

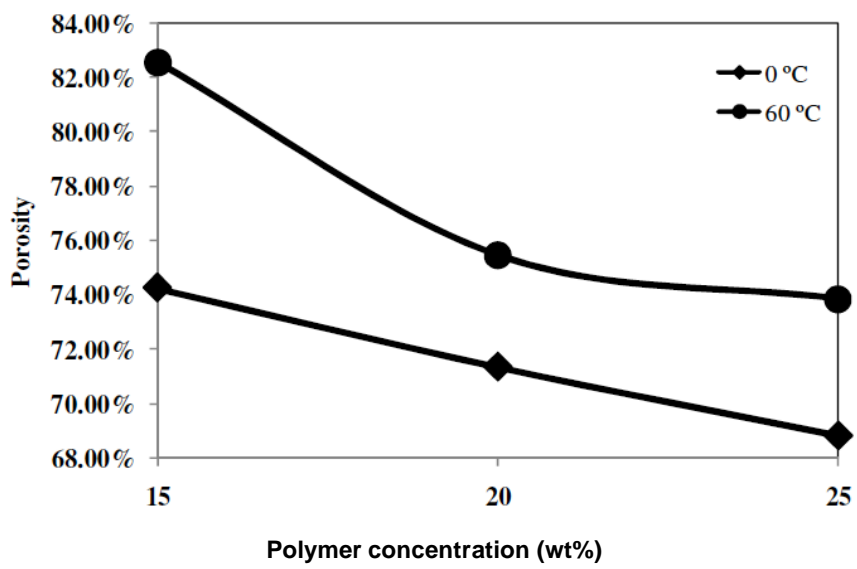

Figure 1: Effect of polymer concentration on membrane porosity, coagulation bath temperature $0^{\circ} \mathrm{C}(-\mathbf{-})$ and 60 ${ }^{\circ} \mathrm{C}(->)$.

Figure 2 shows the SEM images of fabricated HDPE membranes in different polymer concentrations. As shown in the Figure 2, increasing the polymer concentration increases the number of leafy structures of pores and compresses them which resulting in lower porosity. It can be concluded that polymer concentration not only affects on the size of pores but also changes the pores morphologies.

In high polymer concentration, when nucleation density increases, accumulation of crystals increases which results in lower porosity. Compacted leafy structures prevent the spherulites to completely grow in which in high polymer concentrations, leafy structure is mainly observed.

\subsection{The Impact of Coagulation Bath Temperature on the Membrane Porosity}

In membrane fabrication via TIPS method, coagulation bath induces the phase separation and forms the polymer crystals. It can be concluded that 

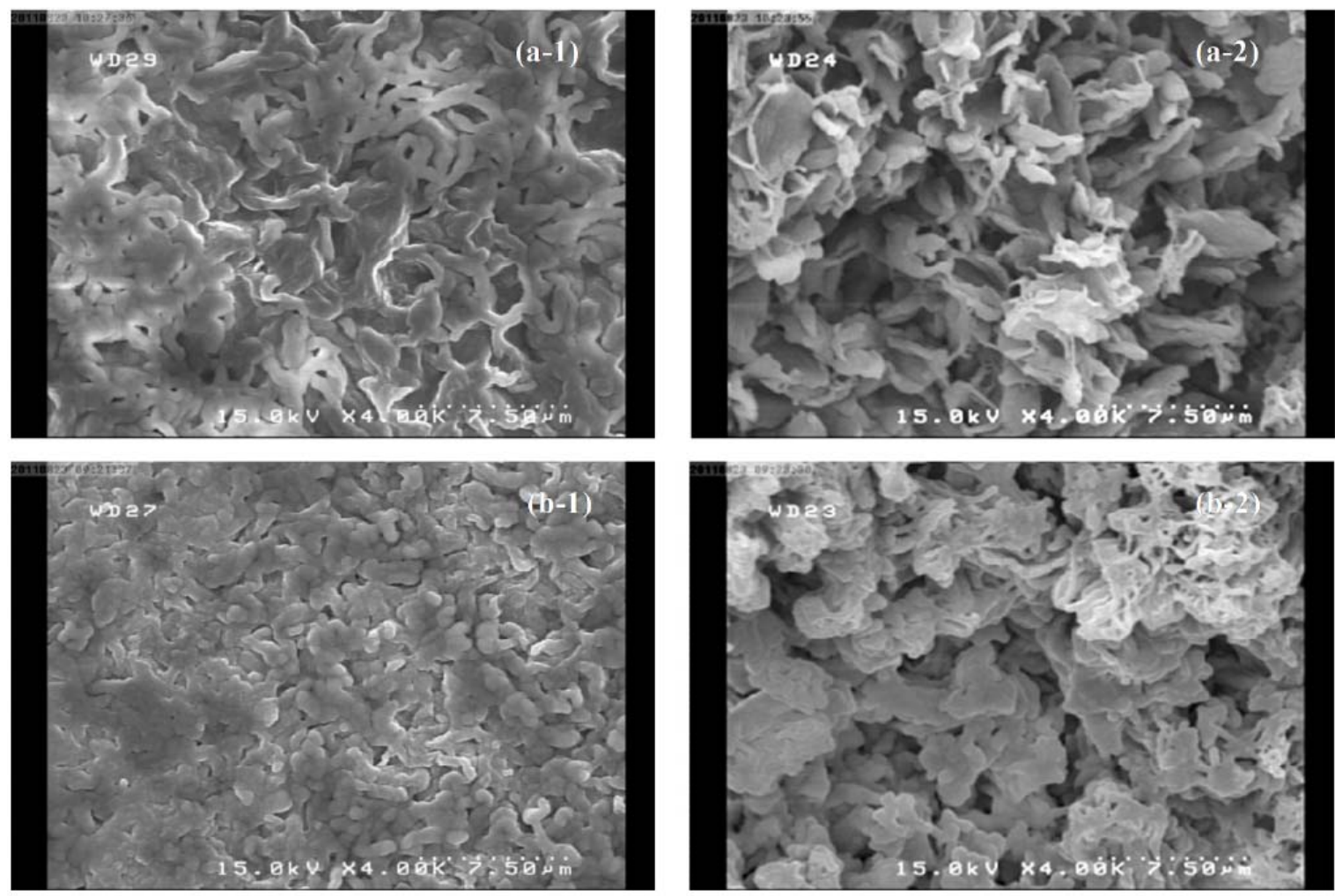

Figure 2: SEM images of prepared membranes in $60{ }^{\circ} \mathrm{C}$ coagulation bath temperature. (a) Polymer concentration = $15 \%(\mathrm{w} / \mathrm{w})$; (b) Polymer concentration $=25 \%(w / w)$. (1) Outer surface; (2) Cross-section.

coagulation bath temperature would also affects on the structure and properties of membranes. The table of data variance analysis of porosity also confirms the remarkable impact of coagulation bath temperature on the membrane porosity. The impact of coagulation bath temperature on the membrane porosity is shown in Figure 3. For all utilized concentrations, increasing of coagulation bath temperature increases the membrane porosity.

When the bath temperature increases, the cooling rate decreases and crystals get enough time to completely grow and leafy structure of HDPE membrane is created. Meanwhile, with increment of the size of polymer crystals, the gap between crystals as well as the membrane porosity increase.

Figure 4 shows the SEM images of the impact of coagulation bath temperature on the fabricated HDPE membrane. It is clearly shown that when the coagulation bath temperature increases, a porous structure in the bulk and outer surface of membrane is obtained.

\subsection{Characterization of Prepared HDPE Membranes}

SEM images of prepared membranes illustrated that no discernible spherulites were exhibited in the tested range of HDPE concentration and leafy structure was mainly formed. Under isothermal crystallization condition for the low molecular weights, crystallization evidently occurs with well-defined crystallographic surface. The diffusion of chain units to the nucleation site is sufficiently facile to allow good crystallographic packing [18]. Therefore for the low molecular weight, spherulite structure is mainly formed. In this structure when the first spherical layer is completed, the second layer builds up around, pointing in the opposite direction, due to space-filling reason. When polymer molecular weight increases, the sheets become shorter, and the amorphous regions between lamellar sheets become thicker. At very high molecular weights, diffusion to the crystallizing surface becomes impeded and rate-controlling. Nucleation appears to occur haphazardly onto the growing crystal face, so that the lamellae are bent and twisted and not organized with each other and leafy structure is formed [19]. In this work HDPE polymer with low MFI (high molecular weight) is used. As shown in SEM images, for the HDPE-LP system, a similar leafy structure was formed. It has been reported that the lamellar-like crystallites can be formed for the linear PE with high molecular weight fraction in melt crystallization $[5,18,19]$. Since in our work, the molecular weight of utilized PE was too much high, similar to the data reported by Voigt-Martin 


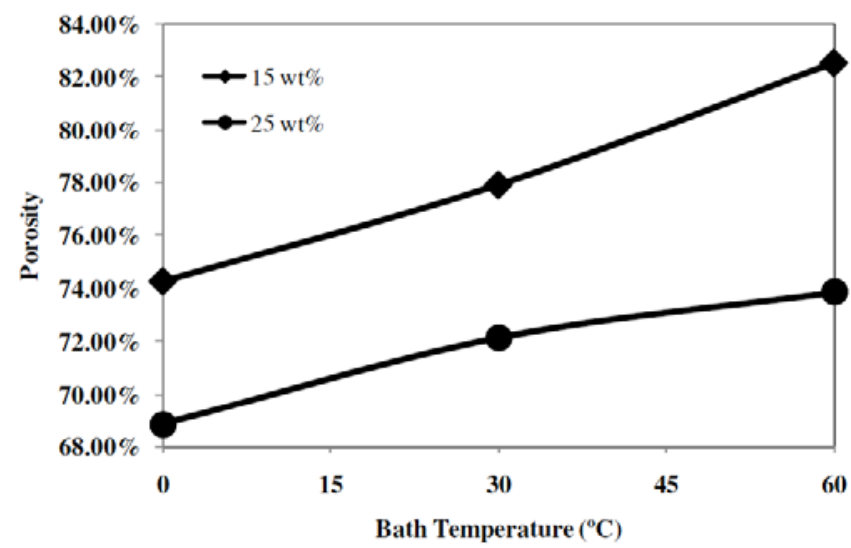

Figure 3: Effect of coagulation bath temperature on membrane porosity, polymer concentration $15 \%(\mathrm{w} / \mathrm{w})(-$ ), polymer concentration $25 \%(\mathrm{w} / \mathrm{w})(-\boldsymbol{-})$.

et al. [18], instead of spherutical structure, leafy structure was mainly obtained.

Another interesting point is the impact of polymer concentration on the membrane structure. The curves in Figure $\mathbf{3}$ show two different slopes for two polymer concentration ranges. In low polymer concentration, when bath temperature varies between 30 and $60^{\circ} \mathrm{C}$, the slope is higher than when bath temperature varies between 0 and $30^{\circ} \mathrm{C}$. The porosity measured in this work is a general index showing porosity in depth and porosity on the surface and therefore this phenomenon could be attributed to the possible formation of spherutical structure and consequently high porosity in low polymer concentrations. On the other hand, when polymer concentration increases, the number of nuclei increases. Increasing of nuclei is corresponded by the increasing of the density of nucleation which increases the probability of nuclei collisions. Therefore, when coagulation bath temperature increases, the tendency for polymer nuclei growth is increased which makes the leaves to be interfused. This phenomenon is clearly shown in SEM images. Therefore in the high coagulation bath temperatures the collision between crystals prevents them to grow completely and the slope of porosity-coagulation bath temperature declines. SEM images in Figures 2 and $\mathbf{4}$ confirm this point.

\section{CONCLUSION}

The results obtained from analysis of variance table using 95 percent confidence interval on the membrane porosity illustrated that the impact of polymer concentration on the membrane porosity is more severe than the coagulation bath temperature. Polymer
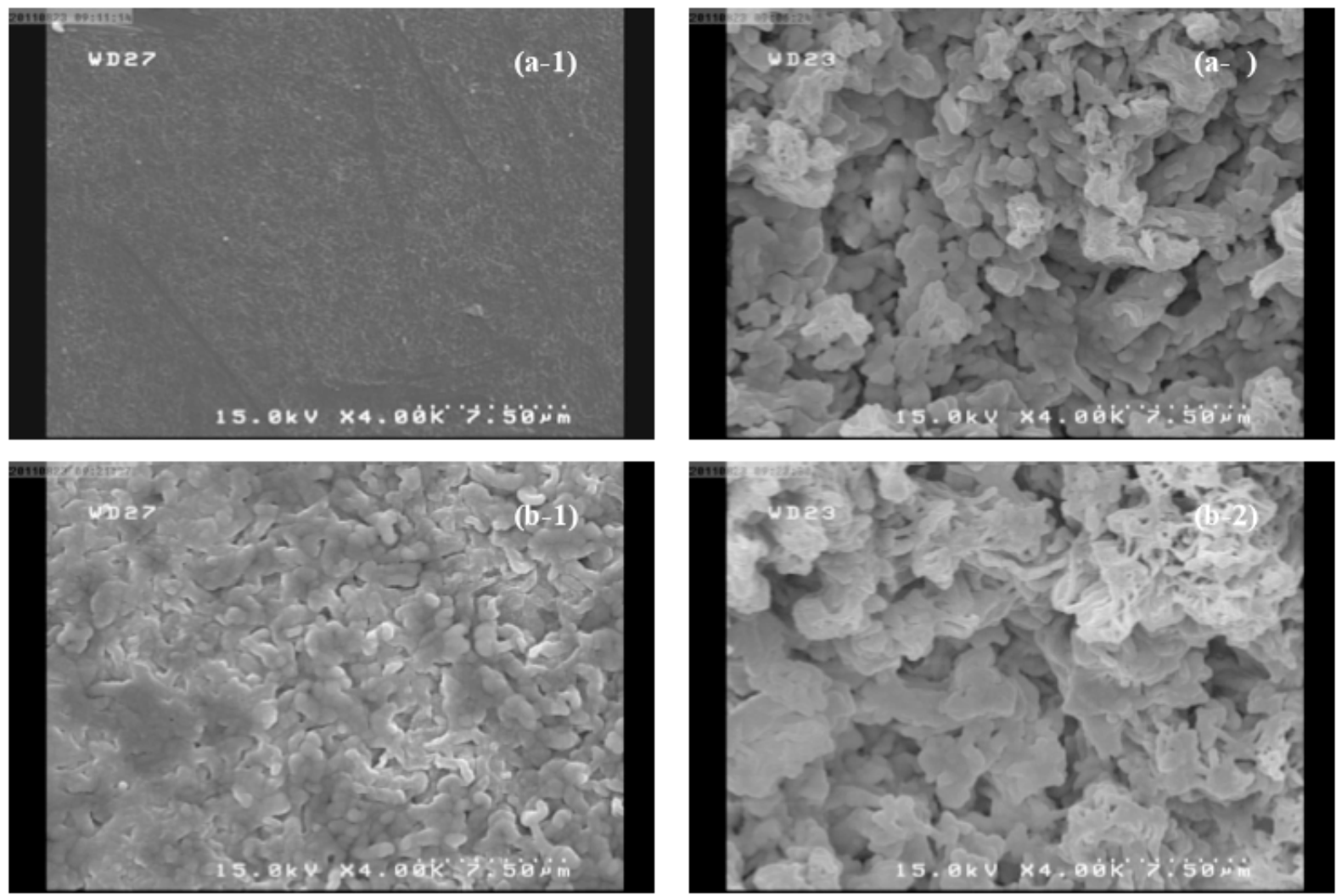

Figure 4: SEM images of 25 wt. \% HDPE in LP. (a) Coagulation bath temperature $=0{ }^{\circ} \mathrm{C}$; (b) $60{ }^{\circ} \mathrm{C}$. (1) Outer surface; (2) Cross-section. 
concentration and membrane porosity are inversely proportionate. On the other hand, increasing the polymer concentration caused to decrease in membrane porosity.

Increasing coagulation bath temperature results in decreasing of cooling rate in which polymers crystals get enough time to completely grow and leafy structure of HDPE membrane is obtained. In the high coagulation bath temperatures the collision between crystals prevents them to grow completely. Therefore the slope of porosity-coagulation bath temperature declines. It was concluded that the polymer molecular weight as well as polymer-diluent ratio not only affects on the membrane porosity but also changes the pore morphology.

\section{REFERENCES}

[1] Sun H, Rhee KB, Kitano T, Mah SI. High-density polyethylene (HDPE) hollow fiber membrane via thermally induced phase separation. I. Phase separation behaviors of HDPE-liquid paraffin (LP) blends and its influence on the morphology of the membrane. J Appl Polym Sci 1999; 73: 2135-42.

http://dx.doi.org/10.1002/(SICl)10974628(19990912)73:11<2135::AID-APP9>3.0.CO;2-X

[2] Mulder M. Basic Principles of Membrane Technology, 2 ed., Kluwer Academic 1996.

[3] Liu S, Zhou C, Yu W. Phase separation and structure control in ultra-high molecular weight polyethylene microporous membrane. J Membr Sci 2011; 379: 268-78. http://dx.doi.org/10.1016/j.memsci.2011.05.073

Rajabzadeh S, Maruyama T, Sotani T, Matsuyama H. Preparation of PVDF hollow fiber membrane from a ternary polymer/solvent/nonsolvent system via thermally induced phase separation (TIPS) method. Separat Purificat Technol 2008; 63: 415-23.

http://dx.doi.org/10.1016/j.seppur.2008.05.027

[5] Lloyd DR, Kinzer KE, Tseng HS. Microporous membrane formation via thermally induced phase separation. I. Solidliquid phase separation. J Membr Sci 1990; 52: 239-61. http://dx.doi.org/10.1016/S0376-7388(00)85130-3

[6] Lloyd DR, Kim SS, Kinzer KE. Microporous membrane formation via thermally-induced phase separation. II. Liquid-liquid phase separation. J Membr Sci 1991; 64: 1-11. http://dx.doi.org/10.1016/0376-7388(91)80073-F

[7] Kim SS, Lloyd DR. Microporous membrane formation via thermally-induced phase separation. III. Effect of thermodynamic interactions on the structure of isotactic polypropylene membranes. J Membr Sci 1991; 64: 13-29. http://dx.doi.org/10.1016/0376-7388(91)80074-G
[8] Lim GBA, Kim SS, Ye Q, Wang YF, Lloyd DR. Microporous membrane formation via thermally-induced phase separation. IV. Effect of isotactic polypropylene crystallization kinetics on membrane structure. J Membr Sci 1991; 64: 3140

http://dx.doi.org/10.1016/0376-7388(91)80075-H

[9] Kim SS, Lim GBA, Alwattari AA, Wang YF, Lloyd DR Microporous membrane formation via thermally-induced phase separation. V. Effect of diluent mobility and crystallization on the structure of isotactic polypropylene membranes. J Membr Sci 1991; 64: 41-53. http://dx.doi.org/10.1016/0376-7388(91)80076-I

[10] Alwattari AA, Lloyd DR. Microporous membrane formation via thermally-induced phase separation. VI. Effect of diluent morphology and relative crystallization kinetics on polypropylene membrane structure. J Membr Sci 1991; 64: 55-67.

http://dx.doi.org/10.1016/0376-7388(91)80077-J

[11] McGuire KS, Lloyd DR, Lim GBA. Microporous membrane formation via thermally-induced phase separation. VII. Effect of dilution, cooling rate, and nucleating agent addition on morphology. J Membr Sci 1993; 79: 27-34.

\section{http://dx.doi.org/10.1016/0376-7388(93)85015-O}

[12] Matsuyama H, Hayashi K, Maki T, Teramoto M, Kubota N. Effect of polymer density on polyethylene hollow fiber membrane formation via thermally induced phase separation. J Appl Polym Sci 2004; 93: 471-74. http://dx.doi.org/10.1002/app.20461

[13] Matsuyama $\mathrm{H}$, Okafuji $\mathrm{H}$, Maki T, Teramoto M, Kubota N. Preparation of polyethylene hollow fiber membrane via thermally induced phase separation. J Membr Sci 2003; 223: 119-26. http://dx.doi.org/10.1016/S0376-7388(03)00314-4

[14] Sun H, Rhee KB, Kitano T, Mah SI. HDPE hollow-fiber membrane via thermally induced phase separation. II. Factors affecting the water permeability of the membrane. $J$ Appl Polym Sci 2000; 75: 1235-42. http://dx.doi.org/10.1002/(SICl)10974628(20000307)75:10<1235::AID-APP4>3.0.CO;2-D

[15] Montgomery DC. Design and Analysis of Experiments, John Wiley \& Sons Australia, Limited 1999.

[16] Shokri E, Yegani R. Full-Factorial experimental design to determine the impacts of influential parameters on the porosity and mechanical strength of LLDEP microporous membrane fabricated via thermally induced phase separation method. J Membr Separat Technol 2012; 1(1): 43-51.

[17] Li X, Wang Y, Lu X, Xiao C. Morphology changes of polyvinylidene fluoride membrane under different phase separation mechanisms. J Membr Sci 2008; 320: 477-82. http://dx.doi.org/10.1016/j.memsci.2008.04.033

[18] Voigt-Martin IG, Fischer EW, Mandelkern L. Morphology of melt-crystallized linear polyethylene fractions and its dependence on molecular weight and crystallization temperature. J Polym Sci Polym Phys Ed 1980; 18: 2347-67. http://dx.doi.org/10.1002/pol.1980.180181204

[19] Wenjun L, Youxin Y, Israel C. Formation and microstructure of polyethylene microporous membranes through thermally induced phase separation. Chin J Polym Sci 1995; 13: 7-19. 